\title{
DIFICULTADES EN EL APRENDIZAJE DE MATEMÁTICAS
}

\section{DIFICCULTIES IN MATHEMATIC LEARNING}

Eliseo Ramírez Rincón ${ }^{1}$

RESUMEN

El artículo corresponde a un avance de la investigación "Comprensión de lenguaje matemático por parte de estudiantes de primer semestre de Ingeniería Agronómica de la U.D.C.A al resolver problemas de matemáticas de variación". Aborda y analiza las dificultades que tuvo un grupo de estudiantes en la clase de matemáticas al realizar un ejercicio sobre una función y sus representaciones.

Palabras clave: Lenguaje, matemáticas, dificultades, función, problemas.

\section{SUMMARY}

This article corresponds to an advance of the research "Comprehension of the mathematical language by students of the first term of U.D.C.A's Agricultural Engineering when solving variation math problems". Tackles and analyzes the difficulties that one group of students had in the mathematics class when realizing an exercise of the function and its representations.

Key words: Language, mathematics, difficulties, function, problems.

\footnotetext{
${ }^{1}$ M.Sc. en docencia de las matemáticas. Estudiante de doctorado. Profesor investigador en Matemática Educativa U.D.C.A elramirez@udca.edu.co; elmatematis@gmail. com (dirección para correspondencia)
}

\section{INTRODUCCIÓN}

D'Amore (2006) indica que son muchos los autores que afirman que la "matemática es por sí misma un lenguaje”. Es evidente esta consideración porque en efecto en las matemáticas hay sintaxis, semántica y pragmática. Las tres se relacionan y no puede haber una sin la existencia de la otra y son éstas las que dan sentido a los objetos de conocimiento matemático, significados y significantes. La comprensión de la sintaxis, la semántica y la pragmática en la enseñanza-aprendizaje de las matemáticas es un proceso lento y, por demás, muy difícil de abordar.

Tanto la enseñanza como el aprendizaje son procesos muy complejos en los que intervienen diferentes actores en un marco cultural y social con la finalidad de poner en discusión: costumbres, valores, problemas, preguntas, conceptos, saberes disciplinares y saberes propios de cada cultura, entre personas que tienen intereses comunes. Estas acciones están mediadas por la comunicación generada a través de los lenguajes (común, de las ciencias, de las matemáticas, del aula, tecnológico,...), de las diversas formas de interacción de las personas y de la ayuda de otras ciencias, como la pedagogía, la didáctica, la tecnología y demás disciplinas que buscan hacer más favorable el tránsito en estos procesos.

El conocimiento de las matemáticas, en general, al ser complejo es multidireccional y, por ende, no se puede reducir ni tampoco linealizar. Los saberes que emanan de éstas y que circulan en la sociedad moderna son prioritariamente de tres formas: el saber disciplinar (matemático), el saber escolar (institucional) y el saber del aula (profesor- estudiante).

Según Godino (2007), el lenguaje como conformador y producto resultante de las mentes individuales ha sido 
y sigue siendo objeto de estudio desde Wittgenstein, Vygotsky hasta el interaccionismo simbólico. La investigación sobre el lenguaje está mostrando que éste tiene una marcada influencia en el proceso de enseñanzaaprendizaje de las matemáticas y que, por lo tanto, es en educación uno de los aspectos a los que se les debe prestar especial interés.

El proyecto sobre "Comprensión del lenguaje matemático", surge por una parte como resultado del proyecto "Errores que cometen los estudiantes de primer semestre de ingenierías de la U.D.C.A, al resolver problemas de matemáticas" (Ramírez, 2006). En el trabajo sobre errores entre otros, se encontró, por ejemplo, que para un mismo objeto de conocimiento matemático los estudiantes no encontraban ninguna relación entre la simbología matemática, el lenguaje común de ellos, los libros y el lenguaje del profesor. Hecho preocupante y de especial interés, porque por una parte los estudiantes consideran que la simbología de las matemáticas es muy difícil y "enredada" para ellos, lo que aparentemente el profesor comunica no es lo que los estudiantes están entendiendo y al recurrir a los textos de consulta la comprensión tampoco es fácil para ellos.

Por otra parte, el creciente interés en el tema mostrado por los grupos de investigación en Educación Matemática como, por ejemplo, PME (Psicología de la Educación Matemática), DME (Didáctica de la Matemática Educativa), quienes consideran que el Lenguaje debe ser un mediador en la comunicación de conocimientos matemáticos y no una dificultad más que deben sortear los estudiantes para aprender matemáticas en el colegio o en la universidad. Lo anterior ha servido de antecedente investigativo para iniciar el proyecto de investigación "Comprensión del Lenguaje Matemático por parte de estudiantes de primer semestre de ingeniería Agronómica de la U.D.C.A". Este artículo presenta un avance del trabajo sobre "Comprensión del Lenguaje Matemático" correspondiente a la tercera fase del proyecto, en la que se propuso estudiar el trabajo individual de los estudiantes en la clase de matemáticas sobre la forma como ellos pasan la información dada en un contexto determinado de un lenguaje a otro (natural o matemático).

Se analiza un ejercicio trabajado con los estudiantes, correspondiente a una función y sus representaciones. La función propuesta fue trabajada por 28 jóvenes de primer semestre de Ingeniería Agronómica de la U.D.C.A, en el segundo semestre de 2006, en dos de las clases regulares, de dos horas cada una. El objetivo pretendía que ellos percibieran las representaciones (tabular, gráfica y algebraica) de la función como equivalentes semánticas de ésta. Sin embargo, respecto a la misma función, las dificultades manifestadas correspondieron al hecho de que ellos entendieron que la representación gráfica obtenida con el programa Graphic 4.1 (Johansen, 2005) era una "verdad" incuestionable y que en cambio las representaciones tabular y algebraica podían estar equivocadas. Llo anterior puede significar, por una parte, que la percepción gráfica es muy potente para ellos y es vista como el mismo objeto matemático en cuestión; al respecto Duval (1999) afirma que para que haya comprensión de un concepto matemático es necesario diferenciar el objeto matemático de sus representaciones.

De otra parte, la comprensión que sobre las representaciones de la función tienen los estudiantes está muy ligada a la concepción que tienen del infinito, visto éste como un conjunto acabado (infinito actual), en el que los sucesos que se perciben (en la representación gráfica) constituyen la misma función, ignorando las otras representaciones como parte del análisis de la misma función, es decir, confunden el objeto con una de sus representaciones.

\section{MATERIALES Y MÉTODOS}

El ejercicio, se propuso inicialmente para que los estudiantes hallaran el dominio, el rango y determinaran la continuidad de la función. El ejercicio, se convirtió en un problema para todos los estudiantes, porque se dividieron las opiniones respecto a la continuidad de la función en $\mathrm{x}=0$. Al principio para todos era evidente dicha discontinuidad y algunos decían que era una discontinuidad asintótica y otros conjeturaron que era removible. En esta discusión, se fueron las dos primeras horas de trabajo de clase y, por lo tanto, se hizo necesario retomar la situación en la siguiente clase. En esta segunda sesión, se utilizó el programa Graphic 4.1 (Johansen, 2005), como ayuda visual y la primera impresión de la gráfica obtenida les causó confusión en los estudiantes en el sentido que esta representación no mostraba en detalle la discontinuidad, sino que por el contrario la gráfica daba la impresión que la función era continua en $\mathrm{x}=0$, por supuesto concluyeron que estaban 
equivocados junto con el profesor al decir que había una discontinuidad en $\mathrm{x}=0$. A partir de este hecho surgió la idea de este trabajo.

\section{RESULTADOS Y DISCUSIÓN}

En el proceso que han seguido los estudiantes a lo largo de su vida académica en cuanto al aprendizaje de las matemáticas es evidente la influencia tanto de lo local (colegio, comunidad, profesores, compañeros,...) como de lo global (políticas educativas, cultura, sociedad, institución matemática,...) y, por lo tanto, la enseñanza y el aprendizaje de las matemáticas dependen de algunos aspectos fundamentales, como los enmarcados en las siguientes preguntas.

¿Qué visiones de las matemáticas tenemos y trasmitimos los profesores de matemáticas?

Para responder a esta pregunta es necesario revisar también la visión que tienen los profesores de matemáticas y de ciencias (particularmente en las naturales por su alto contenido matemático como en física) sobre educación científico (por lo menos en occidente) y sobre educación matemática; desde el punto de vista del desarrollo de pensamiento científico, que permita a cualquier ciudadano poder razonar y comprender aspectos fundamentales de las situaciones que le son cotidianas o que de alguna manera inciden en sus diario vivir, como por ejemplo: el recalentamiento global, el agua como recurso no renovable, el uso de pesticidas, la inflación, el T.L.C., entre otras razones porque las dos disciplinas representan una necesidad del desarrollo social y personal de los individuos y además ambas han estado ligadas en los procesos históricos y epistemológicos del desarrollo humano.

Las expectativas puestas en la contribución de las ciencias a personas que están en sociedades modernas, GilPérez E Vilches (2001), no se han cumplido y en cambio han promovido un fracaso generalizado. Lo que es peor han generado un creciente rechazo de los estudiantes hacia el aprendizaje de las ciencias y, por ende, también de las matemáticas,. Esto, porque la visión de ciencia que ha prevalecido es muy matematizable y la visión que se ha trasmitido de las matemáticas es como un producto acabado y no como una construcción social, en la que los acuerdos de la construcción de los objetos matemáticos corresponde a la comunidad de matemá- ticos y la educación matemática debe ser oficio de los profesores de matemáticas, formados para educar a los jóvenes en los principios, los procesos, las habilidades y las competencias de las matemáticas que les permitan resolver problemas en diversos contextos (matemáticas, de otras ciencias y cotidianos). Lo anterior ha repercutido para que los estudiantes se alejen de las ciencias y de las matemáticas, porque las consideran como de seres especiales o de "locos", aspectos que no corresponden a la realidad de la comunidad de científicos destacados en la historia, como Leibnitz, Newton, Galileo, Einstein, Gauss, Riemann, porque como seres humanos tuvieron dificultades propias de los humanos y sus trabajos correspondieron entre otros a logros obtenidos por muchos otros investigadores, inclusive de épocas distintas.

Algunos estudiosos del problema de la enseñanza de las ciencias, como Furio $E$ Vilches (1997), han mostrado que hay graves distorsiones de la naturaleza de la ciencia que justifican, en gran medida, tanto el fracaso de buen número de estudiantes como su rechazo de la ciencia. Hasta el punto de que hayamos comprendido, como afirman Guilbert $\mathcal{E}$ Meloche (citados en Gil-Pérez $\mathcal{E}$ Vilches, 2004), que la mejora de la educación científica exige, entre los requisitos ineludibles, modificar la imagen de la naturaleza de la ciencia que los profesores tenemos y trasmitimos. El anterior análisis incluye también a la enseñanza de las matemáticas, porque son evidentes las distorsiones de la naturaleza de éstas en la medida en que no se ubican los objetos matemáticos en la historia, se desconocen los problemas que en su momento se pretendían resolver con las matemáticas de esa época, no se establecen relaciones de los alcances que en matemáticas tuvieron algunas culturas (en la historia de la humanidad), las dificultades que padecieron en esta empresa y las diferencias con la actual matemática, no solo desde el rigor si no desde lo epistemológico, la didáctica y su lenguaje.

Estas distorsiones han sido favorecidas y propagadas con ayuda no sólo de lo institucional, sino desde los mismos individuos que de alguna manera pertenecen a esta gran comunidad.

Las investigaciones coinciden en mostrar que la mayoría de los docentes y los estudiantes de matemáticas y de ciencias naturales comparten la creencia de que las matemáticas y el saber científico son "verdaderos". Objetos de conocimiento, conceptos, leyes y teorías... son constitutivos del mundo abstracto y real, en los que 
matemáticos y científicos se limitan a "descubrirlos". El supuesto básico es: existe un mundo real que es cognoscible mediante la razón humana.

En las ciencias naturales, por ejemplo, "Fuerza, inercia, gravedad no son construcciones mentales sino entidades realmente existentes que revelan una estructura causal del mundo y permiten una explicación mediante estos mecanismos causales" (Rodríguez, 1998). En matemáticas, en cambio, los objetos son construcciones mentales que responden a una lógica y se ubican en el marco de una cultura que los avala y son demostrables a través de la deducción y el rigor de las matemáticas, por lo tanto, hay que recordar que dichos objetos no existen en la realidad y que este hecho los convierte ya en una dificultad, porque se puede confundir los objetos con sus representaciones. En este sentido, las investigaciones cognitivas están interesadas en estos procesos relacionados con el aprendizaje de los objetos de conocimiento matemático, donde es fundamental tener en cuenta que la forma en que se aprende no suele coincidir con la manera lógico formal de presentar un objeto matemático ante la comunidad matemática; se puede inclusive afirmar que es frecuente que dicha presentación lógica ofrezca obstáculos cognitivos al estudiante (didácticos, psicológicos o epistemológicos). Las didácticas de las ciencias y las matemáticas no necesariamente deben responder al desarrollo histórico y epistemológico de las disciplinas, sin embargo tampoco se las debe desconocer y por ello los profesores deben tener un excelente nivel en lo disciplinar y un excelente nivel en la didáctica de la disciplina.

Desde la didáctica de las matemáticas un hecho importante lo constituye el trabajo realizado por el grupo de trabajo del ICME 7 (celebrado en Québec, en el año 1992) denominado "Las dificultades de los estudiantes en el Cálculo", que contó con un amplio número de participantes de diferentes países, con el objetivo de responder a algunas cuestiones agrupadas en tres aspectos principales Artigue E Ervynck (1993).

- Objetivos y contenidos: ¿Cuáles son los objetivos de un curso de cálculo?, cंcuál es el papel del cálculo en el currículo de las matemáticas?, ¿cuáles son las relaciones entre los aspectos conceptuales y los aspectos técnicos de los contenidos del curso?

- Dificultades de enseñanza y aprendizaje: ¿Cuáles son las dificultades comunes a todos los aspectos del cálculo?, cicuáles son las dificultades específicas de algunos aspectos?, ¿cuáles son las razones de tales dificultades?

- Concepciones del cálculo y su enseñanza que subyacen en las distintas experiencias: ¿Qué problemas surgen a la hora de implementar secuencias de enseñanza?, cंcuáles han sido los resultados?, cestán de acuerdo los resultados obtenidos con los resultados esperados?, cंes posible explicar las divergencias entre los resultados esperados y los conseguidos?

Muchas de estas preguntas han quedado abiertas y constituyen las preguntas más generales de investigación. Distintos autores han venido señalando un conjunto de dificultades en la enseñanza y en el aprendizaje de los objetos de Análisis Matemático. Se consideran como dificultades esenciales el concepto de límite y los procesos infinitos que intervienen en los conceptos básicos de derivada e integral; se indican además, otro tipo de dificultades que tienen que ver con el estudio de las funciones, la notación de Leibnitz, el concepto de infinito, el uso y selección de las distintas representaciones y el lenguaje de las matemáticas (Ramírez, 2006; Garbin E Azcarate, 2000).

En muchas reformas curriculares, las calculadoras graficadoras y simbólicas y los Programas de Cálculo Simbólico (PCS4) juegan un papel importante. En Estados Unidos, se desarrolla desde 1986 el proyecto C2PC (Calculator and Computer). En Colombia, se ha venido implementando la cultura desde los estándares curriculares como política nacional, inclusive dotando a los colegios de computadores y software especializado, pero eso no es suficiente, porque desde la complejidad de la educación habría que implementar también cambios en la administración del currículo para descentralizar las salas de sistemas y de informática en el sentido que éstas sean laboratorios de trabajo de las ciencias, como la física, la biología, la química y las matemáticas, inclusive las ciencias sociales, por ejemplo, en los modelos económicos se podría ayudar con el ambiente de las salas de informática. Otro hecho a tener en cuenta es la actualización de los profesores en paquetes educativos que faciliten el aprendizaje a través de la simulación, la graficación y la solución de algoritmos.

En matemáticas y en ciencias ċlos profesores tienen una visión acumulativa, de crecimiento lineal? 
Una deformación a la que tampoco suelen hacer referencia los equipos docentes y que es la segunda menos mencionada en la literatura tras la visión exclusivamente analítica, consiste en presentar el desarrollo científico como fruto de un crecimiento lineal, puramente acumulativo (Izquierdo, Sanmarti \& Espinet, (1999) citados en Gil-Pérez $\mathcal{E}$ Vilches (2001).Igualmente en matemáticas en la que prima la axiomatización a partir del pensamiento aristotélico. En ambas visiones, se han ignorando las crisis y las remodelaciones profundas, fruto de procesos complejos que no se dejan ahormar por ningún modelo definido de desarrollo. Esta deformación es complementaria, en cierto modo, de lo que se ha denominado visión rígida, algorítmica, aunque deben ser diferenciadas: mientras la visión rígida o algorítmica se refiere a cómo se concibe la realización de una investigación dada, la visión acumulativa es una interpretación simplificada de la evolución de los conocimientos: matemáticos y científicos, a través de la historia, como fruto de la complejidad de investigaciones realizadas en determinado campo. Una visión simplificada a la que la enseñanza suele contribuir al presentar las teorías y los conceptos hoy aceptadas(os) sin mostrar el proceso de su establecimiento, ni referirse a las frecuentes confrontaciones entre teorías rivales, ni a los complejos procesos de cambio, que incluyen auténticas "revoluciones científicas" (Kuhn, 1970).

Las anteriores preguntas intentan abrir un espacio a la reflexión sobre el hecho que los estudiantes cuando llegan a la universidad, traen consigo unas ideas propias de la ciencia y de las matemáticas y que muchas de ellas tienen el arraigo de las creencias (como acto de fe) que han sido generadas en el ambiente escolar previo, en las raíces culturales, en la sociedad y en la disciplina de cada área del conocimiento.

El siguiente es el ejemplo desarrollado con los estudiantes y con él se pretende mostrar la dificultad que se genera en la comprensión del paso entre las representaciones de la función: gráfica, tabular, algebraica y en lenguaje natural, porque es usual que en el colegio se inicie a los estudiantes en el concepto de función privilegiando una de sus representaciones descuidando las otras. Entre las familias de funciones sucede algo parecido, se favorece a las funciones de naturaleza algebraica y se descuidan las trigonométricas, la exponencial y la logarítmica. El ejercicio propuesto corresponde a la familia de funciones que mejor conocen ellos, la algebraica.

El ejemplo escogido, se recrea en tres de las representaciones de la función, (algebraica, tabular y gráfica), con el fin de mostrar que para los estudiantes en este nivel es usual que consideren a cada representación de la misma función como representaciones de distintas funciones también.

\section{Representación algebraica}

$$
f(x)=\frac{\sqrt{(x+3)}-\sqrt{3}}{x}
$$

En las gráficas 1, A y B de la misma función, se muestran dos registros distintos de la gráfica de la función $\mathrm{f}$. La representación de la gráfica $1 \mathrm{~A}$, muestra la función $\mathrm{f}$, como si fuese continua en $\mathrm{x}=0$, y la de la gráfica $1 \mathrm{~B}$,
A

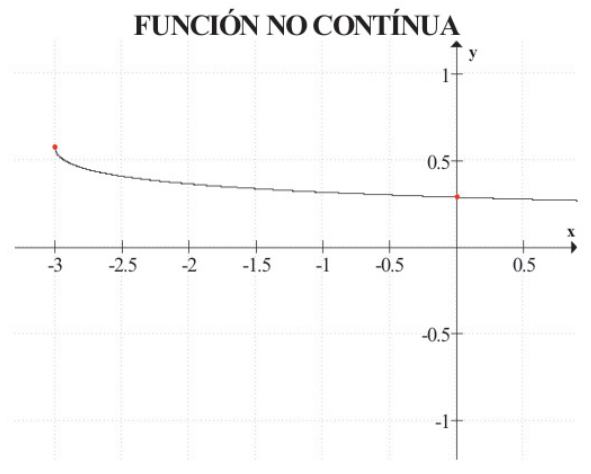

B

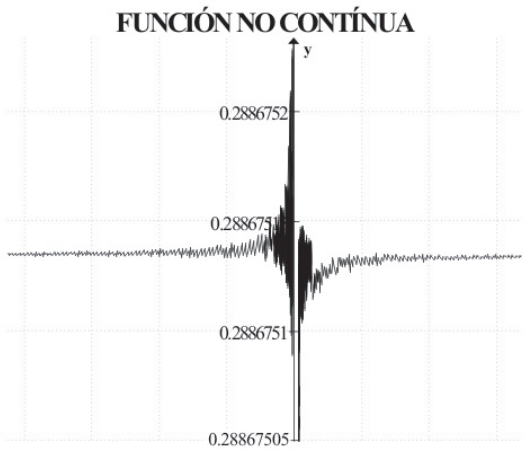

Gráfica 1. Se muestran dos gráficas de la función $\mathrm{f}$. La representación de la A, muestra la función como si fuese continua y la de la B, presenta una discontinuidad en $\mathrm{x}=0$. 
presenta una discontinuidad en $\mathrm{x}=0$, que es el objeto de estudio de este artículo porque en el grupo mencionado se presentó gran dificultad en la comprensión de la función y de sus representaciones.

La tabla 1 corresponde a valores asignados a la función $\mathrm{f}$. Dichos valores están en el intervalo cerrado $[-0,5 ; 0,5]$ en décimas. En esta representación, se presenta la función $\mathrm{f}$, en un intervalo restringido. Esta tabla no proporciona valores de la función ni de sus derivadas en $x=0$, porque la función no existe allí (no existir una función significa que, para un valor asignado a $\mathrm{X}$, su correspondiente $\mathrm{f}(\mathrm{x})$, no tiene valor numérico). La tabla 2 de valores para la función está dada en milésimas en un intervalo cerrado [-0.001, 0.001]. En esta representación, se restringe aún más el intervalo, con el fin de percibir algún cambio en la función en las cercanías de $\mathrm{x}=0$, sin embargo en este intervalo no se percibe.

Tabla 1. Intervalo en décimas de la función; no existe para $x=0$, que es decreciente en $[-5,0) \cup(0,5]$ y cóncava hacia arriba.

\begin{tabular}{|c|c|c|c|}
\hline $\mathbf{x}$ & $\mathbf{f}(\mathbf{x})$ & $\mathbf{f}^{\prime}(\mathbf{x})$ & $\mathbf{f}^{\prime \prime}(\mathbf{x})$ \\
\hline$-0,5$ & 0,3018 & $-0,0288$ & 0,0113 \\
\hline$-0,4$ & 0,2990 & $-0,0277$ & 0,0105 \\
\hline$-0,3$ & 0,2963 & $-0,0267$ & 0,0098 \\
\hline$-0,2$ & 0,2937 & $-0,0258$ & 0,0091 \\
\hline$-0,1$ & 0,2911 & $-0,0249$ & 0,0085 \\
\hline 0 & & & \\
\hline 0,1 & 0,2863 & $-0,0233$ & 0,0075 \\
\hline 0,2 & 0,2840 & $-0,0225$ & 0,0071 \\
\hline 0,3 & 0,2818 & $-0,0219$ & 0,0067 \\
\hline 0,4 & 0,2796 & $-0,0212$ & 0,0063 \\
\hline 0,5 & 0,2776 & $-0,0206$ & 0,0060 \\
\hline
\end{tabular}

Tabla 2. Intervalo de la función en milésimas; no existe en $\mathrm{x}=0$, y se observa además que cuando $\mathrm{x}$, se acerca a cero por izquierda y por derecha la función tiende a estabilizarse en un único valor.

\begin{tabular}{|c|c|c|c|}
\hline $\mathbf{x}$ & $\mathbf{f}(\mathbf{x})$ & $\mathbf{f}^{\prime}(\mathbf{x})$ & $\mathbf{f}^{\prime \prime}(\mathbf{x})$ \\
\hline$-0,001$ & 0,2887 & $-0,0241$ & 0,0080 \\
\hline 0 & & & \\
\hline 0,001 & 0,2887 & $-0,0240$ & 0,0080 \\
\hline
\end{tabular}

En la tabla 3, los valores asignados a la función f están en cienmilésimas en un intervalo cerrado [-0.00001, 0.00001]. Se restringe aún más el intervalo y se percibe que la función se estabiliza en un valor constante f(x) (en la tabla 3, no se escribieron todos los valores). Es decir, que si $x$ tiende al valor cero la función se acerca a 0,2887 tanto por la izquierda de cero como por la derecha del mismo. 
Tabla 3. Intervalos en cienmilésimas; si $x$ tiende al valor cero la función se acerca a 0,2887 tanto por la izquierda de cero como por la derecha del mismo valor $(x=0)$.

\begin{tabular}{|c|c|c|c|}
\hline $\mathbf{x}$ & $\mathbf{f}(\mathbf{x})$ & $\mathbf{f}^{\prime}(\mathbf{x})$ & $\mathbf{f}^{\prime \prime}(\mathbf{x})$ \\
\hline$-0,00001$ & 0,2887 & $-0,0241$ & 0,0081 \\
\hline 0 & & & \\
\hline 0,00001 & 0,2887 & $-0,0241$ & 0,0080 \\
\hline
\end{tabular}

De aquí en adelante el programa con el que estábamos evaluando la función desde su representación gráfica (Graphic 4.1) no aceptó intervalos más pequeños porque su capacidad de redondeo probablemente está dada en cienmilésimas y cantidades más pequeñas las asume como cero.

El inconveniente con esta representación radicó en que la mayoría de los estudiantes no comprendieron el significado de que en la tabla no aparecía ningún valor para $\mathrm{f}(\mathrm{x})$ cuando $\mathrm{x}=0$, y más aún cuando al restringir el intervalo (cienmilésimas) aparecía en la tabla $\mathrm{f}(\mathrm{x})=0$, para $\mathrm{x}=0$. Esto produjo en ellos una gran confusión. En esta experiencia, los estudiantes confiaron completamente en la gráfica (gráfica 1, que fue la primera que ellos lograron hacer), la consideraron única y cuestionaron el hecho de "¿por qué si la gráfica muestra una función continua en $\mathrm{x}=0$, el profesor dice que no lo es?”. Fue evidente que la representación gráfica generó en ellos confianza y no se atrevieron a compararla con la información dada en las tablas ni mucho menos con la información que se podía hallar del análisis a la representación algebraica.

En general, en problemas de este tipo el análisis de la representación algebraica fue el que menos se tuvo en cuenta por parte de los estudiantes por su complejidad. En una de las dos clases, se hizo con ellos el análisis algebraico y a través de éste se pudo ver que la función no existe para valores de " $X$ " menores a menos tres [en lenguaje matemático $\mathrm{x}<(-3)$ ], además que para cualquier valor de $\mathrm{x} \geq(-3)$ la función es positiva y tiene una discontinuidad de tipo agujero o hueco en $\mathrm{x}=0$, $\mathrm{y}$ una asíntota horizontal en y $=0$, (eje $\mathrm{x}$ ).
El análisis algebraico permitió constatar las afirmaciones anteriores de la siguiente forma:

1. La función $\mathrm{f}$, presenta una discontinuidad de tipo agujero o hueco en $\mathrm{x}=0$, es decir, que la función en cercanías de $\mathrm{x}=0$, tanto por la izquierda como por la derecha se estabiliza o tiende a un valor constante, en este caso $\left(\frac{1}{2 \sqrt{3}}\right)$ que en la tabla se muestra como una aproximación a 0,2887.

$$
\begin{aligned}
& \frac{\operatorname{Lim}}{x \rightarrow 0}\left[\frac{\sqrt{x+3}-\sqrt{3}}{x}\right] \text {, racionalizando se tiene que: } \\
& {\left[\frac{\sqrt{x+3}-\sqrt{3}}{x}\right]\left[\frac{\sqrt{x+3}+\sqrt{3}}{\sqrt{x+3}+\sqrt{3}}\right] \text { y simplificando }}
\end{aligned}
$$

(numerador) como una diferencia de cuadrados se tiene que: $\frac{x+3-3}{x[\sqrt{x+3}+\sqrt{3}]}$; por lo tanto simplificando la expresión queda que: $\frac{\operatorname{Lim}}{x \rightarrow 0}\left[\frac{1}{\sqrt{x+3}+\sqrt{3}}\right]$ sacando el límite se tiene que: el límite de la función tiende a $\left(\frac{1}{2 \sqrt{3}}\right)$, cuando $x$ tiende a cero por la izquierda y por la derecha. 
2. En $y=0$ hay una asíntota horizontal, porque si $x$ se hace muy grande, es decir $x$ tiende al infinito entonces $f$ tiende a cero, se acerca al eje "X".

$$
\begin{aligned}
& \frac{\operatorname{Lim}}{x \rightarrow \infty}\left[\frac{\sqrt{x+3}-\sqrt{3}}{x}\right]=\frac{\operatorname{Lim}}{x \rightarrow \infty}\left[\frac{\sqrt{x+3}-\sqrt{3}}{\sqrt{x^{2}}}\right]= \\
& \frac{\operatorname{Lim}}{x \rightarrow \infty}\left[\frac{\sqrt{x+3}}{\sqrt{x^{2}}}\right]-\frac{\operatorname{Lim}}{x \rightarrow \infty}\left[\frac{3}{\sqrt{x^{2}}}\right]= \\
& \frac{\operatorname{Lim}}{x \rightarrow \infty}\left[\sqrt{\frac{x+3}{x^{2}}}\right]-\frac{\operatorname{Lim}}{x \rightarrow \infty}\left[\frac{3}{\sqrt{x^{2}}}\right]= \\
& \frac{\operatorname{Lim}}{x \rightarrow \infty}\left[\sqrt{\frac{x+3}{x^{2}}}\right]-\frac{\operatorname{Lim}}{x \rightarrow \infty}\left[\frac{3}{\sqrt{x^{2}}}\right], \text { aplicando el lím }
\end{aligned}
$$

, aplicando el límite se observa que el primer y segundo términos de la expresión tienden a cero porque el denominador crece más rápido que el numerador. Luego

$\frac{\operatorname{Lim}}{x \rightarrow \infty}\left[\sqrt{\frac{x+3}{x^{2}}}\right]-\frac{\operatorname{Lim}}{x \rightarrow \infty}\left[\frac{3}{\sqrt{x^{2}}}\right]=0$, lo cual indica la

existencia de una asíntota horizontal en el valor $\mathrm{y}=0$.

La dificultad manifestada por los estudiantes al trabajar con los tres registros de la función propuesta (algebraico, tabular y gráfico) fue notoria y este tipo de dificultad es necesario tratarlo con mucho cuidado con ellos, en el sentido de dedicarle mucho tiempo para recrear las diferentes situaciones que se pueden presentar en el análisis de una función con las representaciones, de tal manera que a los estudiantes no les quede la sensación de haber asistido a varios análisis diferentes de distintas funciones también, sino que por el contrario, todo lo hecho correspondió a la misma función propuesta f, a través de sus diferentes representaciones.

Con el trabajo hecho en las dos clases con los estudiantes, se puede concluir entre otras que:

- El proceso de enseñanza aprendizaje de las matemáticas es una construcción social.

- Quien aprende construye significados, no reproduce simplemente lo que lee o lo que se le enseña.
- Comprender algo supone establecer relaciones con otros elementos. Los fragmentos de información aislados son olvidados o resultan inaccesibles a la memoria.

- Todo aprendizaje depende de conocimientos previos.

- El aprendizaje es individual

- La actitud del que aprende favorece o limita el proceso de enseñanza aprendizaje.

- El aprendizaje también es colaborativo.

\section{BIBLIOGRAFÍA}

ARTIGUE, M.; ERVYNCK, G. (eds.).1993. Proceedings of Working Group 3 on Students' Difficulties in Calculus, ICME 7, Quebec, Canada. p.52-60

D’AMORE, B. 2006. Didáctica de la Matemática. Capitulo 8. Matemática, didáctica de la matemática y lenguajes. Editorial Magisterio, Bogotá Colombia, p.251-257.

DUVAL, R. 1999. Registros semióticos y aprendizajes intelectuales. Universidad del Valle, Grupo de Educación Matemática capítulos II y III. p. 32-45.

FURIÓ, C.; VILCHES, A. 1997. Las actitudes del alumnado hacia las ciencias y las relaciones ciencia, tecnología y sociedad. En: del Carmen, L. (Coord.). La enseñanza y el aprendizaje de las ciencias de la naturaleza en la educación secundaria. España. Barcelona: Horsori, p.47-71.

GARBIN, S.; AZCARATE, C. 2000. Esquemas conceptuales e incoherencias con relación al concepto de infinito actual. Educ. Mat. (España). 12(3):2730 .

GIL-PÉREZ, D.; VILCHES, A. 2001. Una alfabetización científica para el siglo XXI. Obstáculos y propuestas de actuación. Investig. Escuela (España). 43:27-37.

GIL-PÉREZ, D.; VILCHES, A. 2004. La contribución de la ciencia a la cultura ciudadana. Cultura y Educación. España. p. 23-35. 
GODINO, J. 2007. Seminario Didáctica de la Matemática DIE-UD. Cap. VI Epistemologías de la Matemática. Bogotá, Colombia, p.29-30

JOHANSEN, I. 2005. Graphic 4.1 Software de uso libre. Disponible desde internet en: http//www.padowan. dk (con acceso 03/06/06)

KUHN, T. 1970. The Structure of Scientific Revolutions, $2^{\mathrm{a}}$ ed, 1970. trad. Esp.: La Estructura de las revoluciones científicas, Madrid, FCE, 1977. p.15-20.
RAMÍREZ R., E. 2006. Errores que cometen los estudiantes de primer semestre de ingenierías al resolver problemas de matemáticas. Rev. U.D.C.A Act.E Div. Cient. (Colombia). 9(2):51-61.

RODRÍGUEZ, G. D. 1998. Ciencia, tecnología y sociedad: Una mirada desde la educación en Tecnología. Rev. Iberoam. Educ. 18:107-143.

Recibido: Febrero 22 de 2007

Aceptado: Mayo 16 de 2007 